\title{
First measurement of the cross-correlation between CMB weak lensing and $\mathrm{X}$-ray emission
}

\author{
G. Hurier ${ }^{1}$, P. Singh ${ }^{2}$, and C. Hernández-Monteagudo ${ }^{1}$ \\ ${ }^{1}$ Centro de Estudios de Física del Cosmos de Aragón (CEFCA), Plaza de San Juan 1, planta 2, 44001 Teruel, Spain \\ e-mail: ghurier@ias.u-psud.fr, ghurier@cefca.es \\ 2 Inter-University Centre for Astronomy and Astrophysics, Ganeshkhind, Post Bag 4, Pune 411007, India
}

Received 10 October 2017 / Accepted 5 March 2019

\begin{abstract}
Since the publication of the results of the Planck satellite mission in 2013, the local and early Universes have been considered to be in tension in respect of the determination of amplitude of the matter density spatial fluctuations $\left(\sigma_{8}\right)$ and the amount of matter present in the Universe $\left(\Omega_{\mathrm{m}}\right)$. This tension can be seen as a lack of massive galaxy clusters in the local Universe compared to the prediction inferred from Planck cosmic microwave background (CMB) best-fitting cosmology. In the present analysis we perform the first detection of the cross-correlation between X-rays and CMB weak lensing at 9.1 $\sigma$. We next combine thermal Sunyaev-Zel'dovich effect, X-rays, and weak-lensing angular auto- and cross-correlation power spectra to determine the galaxy cluster hydrostatic mass bias. We derive $\left(1-b_{\mathrm{H}}\right)=0.71 \pm 0.07$. Considering these constraints, we observe that estimations of $\sigma_{8}$ in the local Universe are consistent with Planck CMB best-fitting cosmology. However, these results are in clear tension with the output of hydrodynamical simulations that favor $\left(1-b_{\mathrm{H}}\right)>0.8$.
\end{abstract}

Key words. cosmological parameters - large-scale structure of Universe - galaxies: clusters: general - galaxies: clusters: intracluster medium - cosmic background radiation

\section{Introduction}

Modern cosmology relies heavily on the observations and analysis of cosmic microwave background (CMB) data. If the standard six parameter $\Lambda$ cold dark matter $(\Lambda C D M)$ model provides a satisfying description of the outcome of the main cosmological probes (Planck Collaboration XIII 2016), the determination of its parameters appears to be in tension when separately considering the CMB angular power spectrum on the one hand, and the abundance of galaxy cluster in the local Universe $(z<1)$ on the other (see, e.g., Salvati et al. 2018, for a recent CMB-galaxy cluster joint analysis).

One current challenge of modern cosmology is thus related to the understanding of the origin of this apparent tension. This could be produced by our lack of knowledge regarding the galaxy cluster mass-observable relations, or by new physics beyond the standard $\Lambda \mathrm{CDM}$ model affecting the structure growth between the CMB last scattering surface and the local Universe (Salvati et al. 2018; Planck Collaboration XX 2014). In this context, several probes can be used to trace the large-scale distribution of matter in the Universe. During their propagation along the line of sight, the CMB photons are affected by several physical processes such as the inverse Compton scattering expressed by the thermal Sunyaev-Zel'dovich effect (Sunyaev \& Zeldovich 1969, 1972; hereafter tSZ), and the CMB gravitational lensing (Blanchard \& Schneider 1987) accounting for the deflections induced by the gravitational potential integrated along the line of sight, $\phi$. The hot electrons causing the tSZ effect are also emitting in the $\mathrm{X}$-ray domain through Bremsstrahlung radiation in such a way that the $\mathrm{tSZ}$ effect, X-ray emission, and gravitational lensing have been powerful sources of cosmological and astrophysical constraints (see, e.g., Planck Collaboration XX 2014; Planck Collaboration XVII 2014).

However, the use of galaxy clusters as cosmological probes often requires the determination of their total mass, a complicated step usually relying on the hydrostatic equilibrium hypothesis. While such mass estimates are known to be biased $\left(M_{\text {hydro }} / M_{\text {true }}=1-b_{\mathrm{H}}\right)$, hydrodynamical simulations favor low values for this bias $\left(b_{\mathrm{H}}<0.2\right)$ (see, e.g., Lau et al. 2013; Biffi et al. 2016). At the same time, on the observational side a significant number of analyses combining X-ray, tSZ, and weak lensing observations have been conducted in the last five years, either based on an object-by-object approach or resorting to stacking algorithms (see, e.g., Hurier \& Angulo 2018; Medezinski et al. 2018; Sereno et al. 2017; Jimeno et al. 2018; Parroni et al. 2017; Okabe \& Smith 2016; Battaglia et al. 2016; Applegate et al. 2016; Smith et al. 2016; Hoekstra et al. 2015; Simet et al. 2015; Israel et al. 2015; von der Linden et al. 2014; Donahue et al. 2014; Gruen et al. 2014; Mahdavi et al. 2013). These analyses obtain a hydrostatic mass bias $b_{\mathrm{H}}>0.3$, which seem to be compatible with the measurement obtained after combining CMB weak lensing and tSZ measurements towards SDSS DR8 redMaPPer (Rykoff et al. 2014) galaxy clusters: $b_{\mathrm{H}}=0.26 \pm 0.07$ (Hurier \& Angulo 2018).

The statistical correlations of these tracers on the sky have also attracted a lot of attention. For instance, the correlation between tSZ-X and tSZ- $\phi$ cross-analyses have been used to set cosmological constraints (see, e.g., Hill \& Spergel 2014; Hurier 2015; Hurier et al. 2015). These measurements present different sensitivities to cosmological parameters and mass-observable 
relations. Consequently, a coherent statistical analysis of $\phi, \mathrm{tSZ}$, and $\mathrm{X}$-ray emission is a powerful tool for identifying the origin of the tension between the CMB and the low redshift Universe.

In the present paper we present the first measurement of the CMB weak lensing and X-ray emission cross-correlation power spectrum. We model this cross-correlation using a halo-model formalism to derive cosmological constraints. Finally, we combine this result with previous studies to derive constraints on the hydrostatic mass bias.

\section{Modeling the tSZ, X-ray, and $\phi$ cross-correlations}

We refer to Hurier (2015) for a detailed modeling of tSZ and $\phi$ cross-power spectra, and to Hurier et al. (2014) for the modeling of the X-ray emission. We relate cosmological parameters to the dark matter halo number per unit of mass and redshift $\frac{\mathrm{d}^{2} \mathrm{~N}}{\mathrm{~d} M \mathrm{~d} V}$ using the mass function from Tinker et al. (2008).

\subsection{Poissonian term}

Using the Limber approximation, we can write the one-halo term as

$C_{\ell}^{\mathrm{A} \times \mathrm{B}-\mathrm{P}}=4 \pi \int \mathrm{d} z \frac{\mathrm{d} V}{\mathrm{~d} z \mathrm{~d} \Omega} \int \mathrm{d} M \frac{\mathrm{d}^{2} N}{\mathrm{~d} M \mathrm{~d} V} W_{\mathrm{A}}^{\mathrm{P}} W_{\mathrm{B}}^{\mathrm{P}}$.

The tSZ contribution can be written as

$W_{\mathrm{tSZ}}^{\mathrm{P}}=Y_{500} y_{\ell}$

where $Y_{500}$ represents the tSZ flux of the clusters within a radius where the matter density equals 500 times the critical density at the clusters' redshift, related to the mass contained in the same volume $\left(M_{500}\right)$ via the scaling law presented in Planck Collaboration XX (2014). In this same expression, $y_{\ell}$ represents the Fourier transform on the sphere of the cluster pressure profile per unit of tSZ flux from Planck Collaboration XX (2014). We consider a generalized Navarro-Frenk-White (GNFW) Universal pressure profile (Arnaud et al. 2010; Planck Collaboration Int. V 2013).

We model the lensing contribution as

$W_{\phi}^{\mathrm{P}}=-2 \psi_{\ell} \frac{\left(\chi^{\prime}-\chi\right) \chi}{\chi^{\prime}}$,

with $\chi$ the comoving distance, $\chi^{\prime}$ the comoving distance to the surface of the last scattering of the CMB, and $\psi_{\ell}$ the 3D lensing potential Fourier transform on the sky. We can express the potential $\psi$ as a function of the density contrast,

$\Delta \psi=\frac{3}{2} \Omega_{\mathrm{m}} H_{0}^{2} \frac{\delta_{3 \mathrm{D}}}{a}$,

with $a$ the Universe scale factor and $\delta$ the density contrast. From this, the lensing contribution reads

$W_{\phi}^{\mathrm{P}}=\frac{3 \Omega_{\mathrm{m}} H_{0}^{2}(1+z)}{c^{2} \ell(\ell+1)} \frac{\left(\chi^{\prime}-\chi\right) \chi}{\chi^{\prime}} \delta_{\ell}$,

where $\delta_{\ell}$ is the Fourier transform of the density contrast profile, $\delta_{3 \mathrm{D}}(u)$, computed as

$\delta_{\ell}=\frac{4 \pi r_{500}}{l_{500}^{2}} \int_{0}^{\infty} \mathrm{d} u u^{2} \delta_{3 \mathrm{D}}(u) \frac{\sin \left(\ell u / \ell_{500}\right)}{\ell u / \ell_{500}}$,

where $u=r / r_{500}$ is the normalized radius of the profile, $\ell_{500}=$ $D_{\mathrm{A}} / r_{500}, D_{\mathrm{A}}$ is the angular diameter distance, and $r_{500}$ is the radius within which the matter density is 500 times the critical density of the Universe.

Finally, the X-ray contribution can be written as

$W_{\mathrm{X}}^{\mathrm{P}}=S_{500} x_{\ell}$

with $S_{500}=\bar{C} L_{500}$, the X-ray count-rate in the $[0.5-2.0] \mathrm{keV}$ energy band of the host halo, $L_{500}$ the unabsorbed X-ray luminosity in the $[0.1-2.4] \mathrm{keV}$ energy range, $\bar{C}$ the average luminosity to count-rate conversion factor described in Hurier et al. (2014), and $x_{\ell}$ the Fourier transform of the X-ray number count profile. To model the $L_{500}-M_{500}$ relation, we used the relation derived by Arnaud et al. (2010) from the REXCESS sample (Böhringer et al. 2007). We considered a polytropic equation of state with a polytropic index of 1.2 to compute the density and the temperature profiles from the pressure profile.

\subsection{Large-scale correlation terms}

We express the large-scale correlations, the two-halo term, contribution as

$C_{\ell}^{\mathrm{A} \times \mathrm{B}-\mathrm{C}}=4 \pi \int \mathrm{d} z \frac{\mathrm{d} V}{\mathrm{~d} z \mathrm{~d} \Omega} W_{\mathrm{A}}^{\mathrm{C}} W_{\mathrm{B}}^{\mathrm{C}} P_{k}$,

with $P_{k}$, the matter power-spectrum computed using CLASS (Lesgourgues 2011).

For the tSZ effect, the CMB weak lensing, and the X-ray count rate we can express the window functions as

$W_{\mathrm{tSZ}}^{\mathrm{C}}=\int \mathrm{d} M \frac{\mathrm{d}^{2} \mathrm{~N}}{\mathrm{~d} M \mathrm{~d} V} Y_{500} y_{\ell} b_{\text {lin }}$,

$W_{\phi}^{\mathrm{C}}=\frac{3 \Omega_{\mathrm{m}} H_{0}^{2}}{c^{2} \ell(\ell+1)}(1+z) \frac{\left(\chi^{\prime}-\chi\right)}{\chi^{\prime} \chi}$,

$W_{\mathrm{X}}^{\mathrm{C}}=\int \mathrm{d} M \frac{\mathrm{d}^{2} \mathrm{~N}}{\mathrm{~d} M \mathrm{~d} V} S_{500} x_{\ell} b_{\text {lin }}$,

where $b_{\text {lin }}$ is the linear bias relating the halo distribution to the overdensity distribution. We considered the bias from Mo \& White (1996), which is realistic on galaxy cluster scales.

We find that X-ray-lensing cross-correlation power spectra evolves as $\Omega_{\mathrm{m}}^{1.8} \sigma_{8}^{6.0} b_{\mathrm{H}}^{1.8}$ for $\ell=200$.

We present in Fig. 1 the power density distribution in the $M_{500}-z$ plane for the X-ray, weak lensing, and tSZ auto- and cross-correlation power spectra at $\ell=200$. We observe that weak-lensing favors higher redshift objects compared to the tSZ effect and X-ray emission. The X-ray and tSZ are highly correlated (at $\simeq 76 \%$ ), while the tSZ effect and the weak lensing one-halo terms are moderately correlated $(\simeq 46 \%)$, and the $\mathrm{X}$-ray emission and the weak lensing one halo-terms show a lower correlation at the level of $\simeq 20 \%$. We refer the reader to Hurier (2015) and Hurier et al. (2015) for a detailed description of the mass and redshift dependence of the CMB weak lensing and X-ray window functions. In the light of Fig. 1, it becomes clear that detecting the X-ray-lensing cross-correlation is particularly challenging, considering the high noise level of Planck CMB weak lensing maps, and the AGN-dominated X-ray sky.

\section{First measurement of the cross-correlation between X-rays and weak-lensing}

We use the ROSAT all-sky survey (RASS) public data ${ }^{1}$, which covers $99.8 \%$ of the sky, including $97 \%$ that has an exposure

1 http://www.xray.mpe.mpg.de/rosat/archive/ 

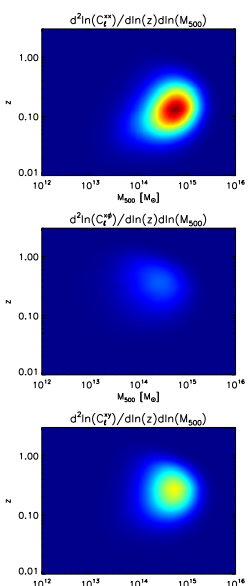

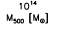
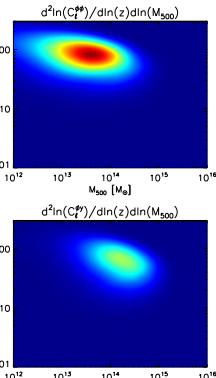

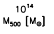

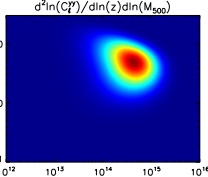

$\frac{10^{14}}{w_{\text {sec }}^{14}\left[w_{\mathrm{s}}\right]}$
Fig. 1. Power density for power spectra $\frac{\mathrm{d}^{2} \ln \left(\mathrm{C}_{\ell}\right)}{\mathrm{d} \ln \left(M_{500}\right) \mathrm{d} \ln (z)}$ as a function of halo mass $M_{500}$ and redshift $z$ at $\ell=200$. On the diagonal we display power density for the auto-correlation power spectra. From left to right: contribution to $\mathrm{X}$-ray, $\mathrm{CMB}$ weak lensing, and $\mathrm{tSZ}$ angular power spectrum at $\ell=200$. Off-diagonal panels represent power density of cross-power spectra, from left to right and top to bottom referring to: $\mathrm{X}$-ray-lensing, X-ray-tSZ, and lensing-tSZ cross-power spectra. The color scale is the same for all panels and represents the total amount of correlation between probes.

time longer than $100 \mathrm{~s}$ (Voges et al. 1999). A description of the reprojection of the RASS data on the Healpix full-sky map can be found in Hurier et al. (2015). We also use the Planck full-sky CMB weak lensing map (Planck Collaboration XVII 2014). We mask all regions with less than $100 \mathrm{~s}$ exposure time in RASS full-sky map, while using the mask associated with the Planck all-sky weak lensing map. This combination of masks results in an effective sky fraction of $f_{\text {sky }} \simeq 75 \%$ in the cross-correlation. We have also verified that using more aggressive galactic masks (down to a sky coverage of $50 \%$ ) does not modify the results significantly. limit as

We compute the uncertainty in the weakly non-Gaussian

$V\left(C_{\ell}^{x \phi}\right)=\frac{\left(C_{\ell}^{x \phi}\right)^{2}+C_{\ell}^{x x} C_{\ell}^{\phi \phi}}{(2 \ell+1) f_{\text {sky }}}$,

where $C_{\ell}^{x \phi}, C_{\ell}^{x x}$, and $C_{\ell}^{\phi \phi}$ are the X-ray-lensing, X-ray, and weak lensing power spectra. We obtained a significance of $9.1 \sigma$ for the X-ray-weak lensing cross-power spectrum in the range $\ell \in$ $[10,400]$.

We verified that AGNs do not produce a significant contribution to the total signal by masking BOSS AGNs (Albareti et al. $2017)$ and performing our analysis on the BOSS footprint $\left(f_{\text {sky }}=\right.$ $25 \%$ ). We also masked NVSS bright sources, $S>0.03 \mathrm{Jy}$ (Condon et al. 1998), and restrict our analysis to the NVSS footprint $\left(\delta>-40^{\circ}\right)$. We do not observe any significant modification of our results when masking the BOSS AGNs or NVSS sources. We also modeled the AGN contribution following the AGN mass and luminosity functions from Hütsi et al. (2014) and Main et al. (2017). This contribution is about two orders of magnitude smaller than the X-ray- $\phi$ cross-correlation, as shown in Fig. 2 for the Hütsi et al. (2014) model. Consequently, the AGN impact on our results is small compared to the uncertainty level. We thus neglect the AGN contribution to the total X-ray-lensing cross-correlation.

In Fig. 2, we present the derived angular cross-power spectrum, compared to our modeling for $\left(\sigma_{8}=0.8, \Omega_{\mathrm{m}}=0.3\right.$,

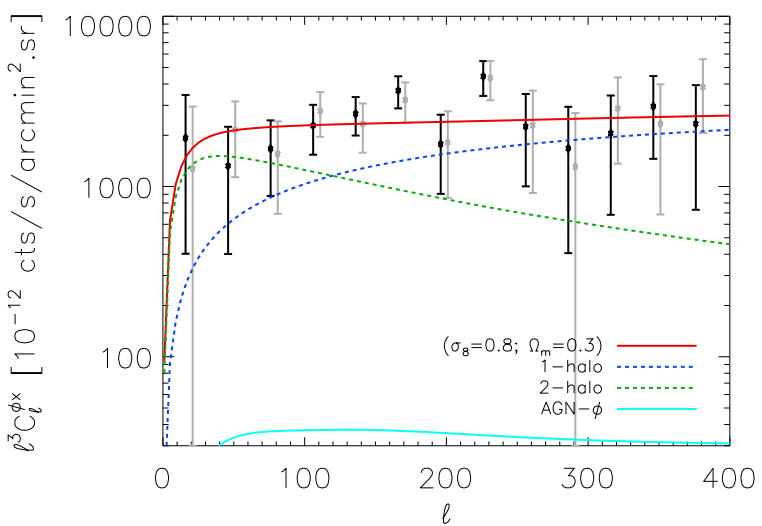

Fig. 2. X-ray- $\phi$ cross-correlation angular power spectrum, measured between Planck CMB lensing full-sky map and a RASS data reprojected on the full-sky (black sample); the same cross-spectra when masking NVSS sources is shown as grey samples. The red solid line shows the theoretical prediction assuming $\left(\sigma_{8}=0.8, \Omega_{\mathrm{m}}=0.3\right.$, $b_{\mathrm{H}}=0.2$ ), the green dashed line shows the two-halo term contribution, and the blue dashed line the one-halo term contribution. The solid cyan line displays the contribution from AGN- $\phi$ cross-correlation.

$b_{\mathrm{H}}=0.2$ ). We observe that the two-halo term dominates for $\ell<100$ and that the one-halo term dominates at higher multipoles. This illustrates how Planck weak lensing map contains a significant signal produced by compact objects like galaxy clusters. Assuming a Gaussian prior of $b_{\mathrm{H}}=0.20 \pm 0.05$, consistent with hydrodynamical simulations and marginalizing over other cosmological parameters according to Planck CMB bestfitting cosmology (Planck Collaboration XIII 2016), we derive $\Sigma_{8}=\sigma_{8}\left(\Omega_{\mathrm{m}} / 0.30\right)^{0.27}=0.80 \pm 0.03$. We verified that masked galaxy clusters only marginally affect $(<1 \%)$ the constraints on $\sigma_{8}$, which can thus be safely neglected.

\section{Combined analysis of thermal Sunyaev-Zel'dovich effect, X-ray, and weak-lensing signals}

We next combine our results with tSZ- $\phi$, tSZ-tSZ, tSZ-Xray, $\phi-\phi$, and $\mathrm{CMB}-\mathrm{CMB}$ power spectra results. We assume that the CMB angular power spectrum is uncorrelated with all other probes ${ }^{2}$, so we compute the covariance between all large-scale structure tracers following the procedure described in Hurier \& Lacasa (2017) accounting for non-Gaussian contributions to the uncertainties. We use the tSZ analysis from Hurier \& Lacasa (2017), the tSZ- $\phi$ measurement corrected for cosmic infra-red background contamination from Hurier (2015), the tSZ-X-ray results from Hurier et al. (2015), the Planck weak lensing results from Planck Collaboration XVII (2014), and the Planck CMB results from Planck Collaboration XIII (2016).

We performed a join fit of these results to derived cosmological constraints on $\sigma_{8}$ and $\Omega_{\mathrm{m}}$ and constraints on the hydrostatic mass bias $b_{\mathrm{H}}$. Both weak-lensing and $\mathrm{CMB}$ results are not sensitive to the hydrostatic mass bias. Consequently, weak lensing and CMB constraints set the cosmological parameters $\sigma_{8}$ and $\Omega_{\mathrm{m}}$, whereas the large-scale structure tracers, namely the

2 This is a reasonable approximation provided the low level of correlation induced by the integrated Sachs-Wolfe effect, which is restricted to very low multipoles $(\ell<50)$. We are also assuming that residual AGN and galaxy cluster tSZ residual contamination on the CMB should be of relevance at much higher multipoles than those considered here $(\ell>1000)$. 


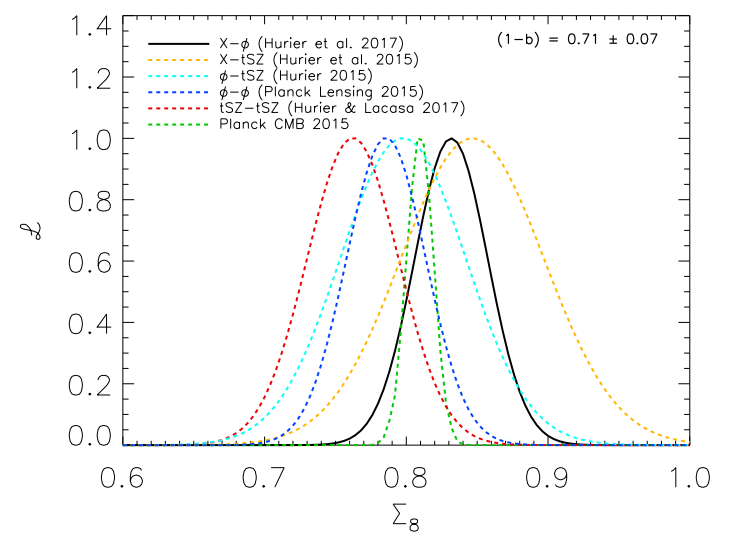

Fig. 3. Likelihood function of $\Sigma_{8}=\sigma_{8}\left(\Omega_{\mathrm{m}} / 0.30\right)^{0.27}$ from different analyses: tSZ angular power spectrum, bispectrum, and number count (red, Hurier \& Lacasa 2017); CMB weak lensing (dark blue, Planck Collaboration XVII 2014); CMB angular power spectrum (green, Planck Collaboration XIII 2016); tSZ-weak lensing cross correlation (cyan, Hurier 2015); tSZ-X-ray cross-correlation (orange, Hurier et al. 2015); and X-ray- $\phi$ cross-correlation (black, this work).

tSZ effect and the X-ray emission, set the hydrostatic mass-bias value. From this combined analysis, we derived $b_{\mathrm{H}}=0.71 \pm 0.07$. We present the resultant likelihoods for $\mathrm{tSZ}, \mathrm{tSZ}-\mathrm{X}, \mathrm{tSZ}-\phi$, $\mathrm{X}-\phi, \phi$, and $\mathrm{CMB}$ analyses for $b_{\mathrm{H}}=0.71 \pm 0.07$ in Fig. 3. The $\mathrm{X}$-ray auto-correlation power spectrum is not shown in this figure as it is particularly difficult to derive robust constraints from it, considering that the X-ray sky is dominated by AGN contribution. We observe that some tension remains, especially with tSZ derived constraints, but all large-scale structure analyses presented here are consistent within $2 \sigma$ with the Planck CMB results.

\section{Conclusion and discussion}

We produced the first detection of the X-ray- $\phi$ crosscorrelation angular power spectrum, with a significance of $9.1 \sigma$. We established cosmological constraints on $\sigma_{8}$ and $\Omega_{\mathrm{m}}$ from this cross-correlation, which we find consistent with previous large-scale structure (Hurier 2015; Hurier et al. 2015; Hurier \& Lacasa 2017; Hurier \& Angulo 2018) and CMB analyses (Planck Collaboration XIII 2016; Planck Collaboration XVII 2014). Similarly to the tSZ-X cross-correlation, the $\mathrm{X}-\phi$ correlation favors values of the hydrostatic mass bias lower than those suggested in tSZ-CMB combined analyses (Salvati et al. 2018). It also favors a higher value for $b_{\mathrm{H}}$ than most of the weak-lensing based analyses of the last four years (see, e.g., Medezinski et al. 2018; Sereno et al. 2017; Jimeno et al. 2018; Parroni et al. 2017; Okabe \& Smith 2016; Battaglia et al. 2016; Applegate et al. 2016; Smith et al. 2016; Hoekstra et al. 2015; Simet et al. 2015; Israel et al. 2015; Donahue et al. 2014; Gruen et al. 2014; Mahdavi et al. 2013). These analyses prefer $b_{\mathrm{H}} \simeq 0.20$. With the constraint inferred here $\left(b_{\mathrm{H}}=0.29 \pm 0.07\right)$, large-scale structure cosmological constraints from the local Universe on $\sigma_{8}$ and $\Omega_{\mathrm{m}}$ now surround the CMB-based cosmological constraints. This result favors a high value of $b$ compared to hydrodynamical simulations that prefer $b_{\mathrm{H}}<0.2$. Additionally, under the assumption that these measurements are systematic-free, the significant difference between tSZ and X-ray based results may indicate that these two probes select significantly different populations of galaxy clusters in terms of hydrostatic mass bias.

Acknowledgements. We acknowledge the use of HEALPix (Górski et al. 2005).

\section{References}

Albareti, F. D., Allende Prieto, C., Almeida, A., et al. 2017, ApJS, 233, 25 Applegate, D. E., Mantz, A., Allen, S. W., et al. 2016, MNRAS, 457, 1522 Arnaud, M., Pratt, G. W., Piffaretti, R., et al. 2010, A\&A, 517, A92 Battaglia, N., Leauthaud, A., Miyatake, H., et al. 2016, JCAP, 8, 013 Biffi, V., Borgani, S., Murante, G., et al. 2016, ApJ, 827, 112 Blanchard, A., \& Schneider, J. 1987, A\&A, 184, 1

Böhringer, H., Schuecker, P., Pratt, G. W., et al. 2007, A\&A, 469, 363 Condon, J. J., Cotton, W. D., Greisen, E. W., et al. 1998, AJ, 115, 1693 Donahue, M., Voit, G. M., Mahdavi, A., et al. 2014, ApJ, 794, 136 Górski, K. M., Hivon, E., Banday, A. J., et al. 2005, ApJ, 622, 759 Gruen, D., Seitz, S., Brimioulle, F., et al. 2014, MNRAS, 442, 1507 Hill, J. C., \& Spergel, D. N. 2014, JCAP, 2, 030

Hoekstra, H., Herbonnet, R., Muzzin, A., et al. 2015, MNRAS, 449, 685 Hurier, G. 2015, A\&A, 575, L11

Hurier, G., \& Angulo, R. E. 2018, A\&A, 610, L4

Hurier, G., \& Lacasa, F. 2017, A\&A, 604, A71

Hurier, G., Aghanim, N., Douspis, M., \& Pointecouteau, E. 2014, A\&A, 561, A143

Hurier, G., Douspis, M., Aghanim, N., et al. 2015, A\&A, 576, A90

Hütsi, G., Gilfanov, M., \& Sunyaev, R. 2014, A\&A, 561, A58

Israel, H., Schellenberger, G., Nevalainen, J., Massey, R., \& Reiprich, T. H. 2015 , MNRAS, 448, 814

Jimeno, P., Diego, J. M., Broadhurst, T., De Martino, I., \& Lazkoz, R. 2018, MNRAS, 478, 638

Lau, E. T., Nagai, D., \& Nelson, K. 2013, ApJ, 777, 151

Lesgourgues, J. 2011, ArXiv e-prints [arXiv:1104 .2932]

Mahdavi, A., Hoekstra, H., Babul, A., et al. 2013, ApJ, 767, 116

Main, R. A., McNamara, B. R., Nulsen, P. E. J., Russell, H. R., \& Vantyghem, A. N. 2017, MNRAS, 464, 4360

Medezinski, E., Battaglia, N., Umetsu, K., et al. 2018, PASJ, 70, S28

Mo, H. J., \& White, S. D. M. 1996, MNRAS, 282, 347

Okabe, N., \& Smith, G. P. 2016, MNRAS, 461, 3794

Parroni, C., Mei, S., Erben, T., et al. 2017, ApJ, 848, 114

Planck Collaboration XIII. 2016, A\&A, 594, A13

Planck Collaboration XVII. 2014, A\&A, 571, A17

Planck Collaboration XX. 2014, A\&A, 571, A20

Planck Collaboration Int. V. 2013, A\&A, 550, A131

Rykoff, E. S., Rozo, E., Busha, M. T., et al. 2014, ApJ, 785, 104

Salvati, L., Douspis, M., \& Aghanim, N. 2018, A\&A, 614, A13

Sereno, M., Covone, G., Izzo, L., et al. 2017, MNRAS, 472, 1946

Simet, M., Battaglia, N., Mandelbaum, R., \& Seljak, U. 2015, Am. Astron. Soc. Meet. Abstr., 225, 443.04

Smith, G. P., Mazzotta, P., Okabe, N., et al. 2016, MNRAS, 456, L74

Sunyaev, R. A., \& Zeldovich, Y. B. 1969, Nature, 223, 721

Sunyaev, R. A., \& Zeldovich, Y. B. 1972, Comments Astrophys. Space Phys., 4, 173

Tinker, J., Kravtsov, A. V., Klypin, A., et al. 2008, ApJ, 688, 709

Voges, W., Aschenbach, B., Boller, T., et al. 1999, A\&A, 349, 389

von der Linden, A., Mantz, A., Allen, S. W., et al. 2014, MNRAS, 443, 1973 